Published in final edited form as:

Annu Rev Med. 2011 ; 62: 233-247. doi:10.1146/annurev-med-070909-182917.

\title{
MECHANISMS OF ENDOCRINE RESISTANCE IN BREAST CANCER
}

\author{
C Kent Osborne and Rachel Schiff \\ Dan L. Duncan Cancer Center, Lester \& Sue Smith Breast Center, Departments of Medicine and \\ Molecular and Cellular Biology, Baylor College of Medicine, One Baylor Plaza, BCM 600, \\ Houston, TX 77030, USA
}

C Kent Osborne: kosborne@bcm.edu; Rachel Schiff: rschiff@bcm.edu

\begin{abstract}
The estrogen receptor (ER) pathway plays a pivotal role in breast cancer development and progression. Endocrine therapy to block the ER pathway is highly effective, but its usefulness is limited by common intrinsic and acquired resistance. Multiple mechanisms responsible for endocrine resistance have been proposed and include deregulation of various components of the ER pathway itself, alterations in cell cycle and cell survival signaling molecules, and the activation of escape pathways that can provide tumors with alternative proliferative and survival stimuli. Among these, increased expression or signaling of growth factor receptor pathways, especially the EGFR/HER2 pathway, has been associated with both experimental and clinical endocrine therapy resistance. New treatment combinations targeting ER and growth factor receptor signaling which block the crosstalk between these pathways and eliminate escape routes have been proven highly effective in preclinical models. Results of recent clinical studies, while partly supporting this approach, also highlight the need to better identify a priori the appropriate patients whose tumors are most likely to benefit from these specific co-targeting strategies.
\end{abstract}

\section{Keywords}

Estrogen receptor; Endocrine therapy; growth factor receptor signaling; crosstalk; combination therapy

\section{INTRODUCTION}

The term endocrine therapy is given to those treatments which target the estrogen receptor (ER) by blocking receptor binding with an antagonist or by depriving the tumor of estrogen. The ER, which has nuclear (genomic) and non-nuclear (non-genomic) functions, is the major driver in the majority of breast cancers. It is expressed in $75 \%$ of breast cancers overall, with its detection being slightly more frequent in tumors from postmenopausal women and less in younger women (1). ER expression is related to patient age and correlates with lower tumor grade, lower tumor proliferation, less aneuploidy, less frequent amplification of the c-erbB2 (HER2) oncogene and concomitant loss of the p53 tumor suppressor gene, expression of progesterone receptor (PR), soft tissue and bone metastases, and slower rates of disease recurrence (1-3). It is not related to initial nodal metastases and thus it does not correlate with long-term disease recurrence and death after primary therapy (3).

These clinical factors, along with ER expression itself, are used to make treatment decisions in patients, especially those with metastatic disease. In some cases, multigene tests are performed on the primary breast tumor to assist in adjuvant therapy decision making and to distinguish which patients might benefit most from a combination of endocrine therapy plus 
chemotherapy, rather than endocrine therapy alone. The 21-gene and 70-gene profiles can classify ER+ tumors according to their aggressiveness, risk of recurrence, and likelihood of benefitting from adjuvant endocrine or chemotherapy $(4,5)$. The stratification of ER+ tumors on this basis indicates that some tumors are more resistant to endocrine therapy than others, despite expressing ER. In general, tumors with high levels of ER and PR, negative for HER2 amplification, slowly proliferating, lower grade histologically and with low risk 21-gene or 70-gene profile scores are more likely to benefit from endocrine therapy and less likely to benefit, if at all, from chemotherapy. ER+ tumors which are more aggressive, morphologically and genetically, are less likely to benefit from endocrine therapy, although there are exceptions. Additional recent molecular profiling studies have stratified ER+ tumors into luminal A and luminal B subtypes. The more aggressive and less endocrine sensitive versus the more indolent and endocrine responsive tumors largely overlap with the luminal B versus the luminal A subtypes. Currently, however, no tests exist which can predict resistance to endocrine therapy with certainty, although tumors with absent ER and PR rarely benefit. Most patients with ER-positive tumors are, therefore, treated with endocrine adjuvant therapy, while cases of ER-positive metastatic disease are treated with endocrine therapy initially and serially until the tumor demonstrates independence from estrogen.

Endocrine therapy is the most effective treatment for ER+ metastatic breast cancer, but its effectiveness is limited by high rates of de novo resistance and resistance acquired during treatment. Only about $30 \%$ of patients with metastatic disease have objective regression of tumor with initial endocrine treatment, while another $20 \%$ have prolonged stable disease. Thus, ER is not the only survival pathway driving most of these tumors, and escape pathways when ER is targeted are already functioning or begin to function during treatment.

Understanding the pathways responsible for resistance in the metastatic setting may provide important clues to the mechanisms of resistance to adjuvant endocrine therapy given before or after primary surgery to eradicate distant micrometastases. Treatment in this setting is much more effective with reductions in the risk of recurrence as high as $60 \%$ with estrogen deprivation therapies using aromatase inhibitors in postmenopausal women $(6,7)$. Unfortunately, biopsying patients with metastatic disease in the lung, bone, or liver is difficult and can be associated with high morbidity rates. However, such tissue is crucial for the molecular profiling of resistant tumors in order to understand escape pathways. Despite these challenges, progress is being made in understanding potential mechanisms of resistance. These come largely from preclinical models of endocrine resistance as well as a greater understanding of the molecular mechanisms by which estrogen works to stimulate the growth of the tumor.

\section{MECHANISM OF ESTROGEN ACTION}

All biological networks have similar characteristics. In order to provide important functions in normal cells under a variety of conditions and stress factors, as well as to keep the cell alive, these networks must be complex with multiple levels of regulation, fine tuning capabilities, redundancy, and evolvability. Collectively these features allow the cell to adapt to cellular stress, toxins, and potentially hostile environments. Cancer cells exploit these "normal functions," which are often altered genetically during oncogenesis, to provide them with a survival advantage and the ability to escape the effects of treatment. The ER signaling pathway is an example of a complex biological pathway that controls a variety of functions such as cell proliferation, apoptosis, and angiogenesis which are exploited by breast cancer cells to serve as a major survival pathway driven by the female hormone estrogen (Fig. 1). 
The classic function of ER is its nuclear function, also referred to as genomic activity, to alter the expression of genes important for normal cellular function and tumor growth and survival. ER modulates the expression of hundreds of genes, some by upregulation of expression and others by downregulation (8). Upon binding to estrogen, ER dimerizes with another receptor monomer and attracts a complex of coactivators and corepressors to specific sites on DNA $(3,9)$. ER can also bind to other transcription factors such as AP-1 and SP-1 at their specific sites on DNA, thereby functioning as a coregulator $(3,10)$. Coregulators serve as a fine tuning mechanism by increasing or reducing the transcriptional activity of the receptor (11). Several coregulators have been implicated in cancer, most notably AIB1 (SRC-3), which is gene amplified in a small percentage--but overexpressed in two-thirds--of all breast cancers. Overexpression of this gene has been implicated in tamoxifen resistance (12).

The ER signaling pathway is also regulated by membrane receptor tyrosine kinases including epidermal growth factor receptor (EGFR), HER2, and insulin-like growth factor receptor (IGF1-R) (13). These membrane kinases activate signaling pathways that eventually result in phosphorylation of ER as well as its coactivators and corepressors at multiple sites to influence their specific functions (13-16). This activation of ER by growth factor receptor signaling is sometimes referred to as ligand independent receptor activation. Crosstalk between the growth factor receptor and ER pathways has been established through several other mechanisms as well. Estrogen can increase the expression of ligands such as transforming growth factor-a (TGFa) and IGF1 (10, 17-19) which can then activate the growth factor receptor pathway $(13,18,20)$. On the other hand, estrogen signaling downregulates the expression of EGFR and HER2 while increasing the expression of IGF1$\mathrm{R}$ (21-23). Activation of the PI3K/AKT and the p42/44 mitogen-activated protein kinases (MAPK) pathways by these receptors, in turn, downregulates the expression of ER and PR (24-29). Thus, while these receptor tyrosine kinases can activate the transcriptional function of ER, they can also reduce estrogen dependence by downregulating the expression of ER, perhaps contributing to the relative resistance to endocrine therapies in tumors amplified for HER2 $(25,30)$.

Studies also suggest that ER may work by non-transcriptional mechanisms. Low levels of ER have been found outside the nucleus in the membrane, cytoplasm, or even in the mitochondria, although the exact location for this receptor remains controversial (31). Some of the nongenomic action of estrogen appears to be too rapid for a transcriptional effect to activate growth factor receptor signaling, including the PI3K/AKT and the Ras/p42,44 MAPK pathways (13). Thus, ER-through this nongenomic activity-can alter the expression of genes normally regulated by growth factors $(13,31,32)$. Finally, the stress kinase pathway via 338 and JNK can also modulate ER function by phosphorylation of ER and its coregulators $(33,34)$. The microenvironment and its associated integrin signaling may exert a similar activity (35). Thus, ER activity and signaling is modulated by a variety of pathways which could also contribute to resistance to ER-targeted therapies, especially when the pathways display aberrant activity in a cancer cell.

\section{MECHANISM OF ACTION OF ENDOCRINE THERAPIES}

Various endocrine therapies work by different mechanisms to antagonize the growth promoting activity of estrogen. Selective estrogen receptor modulators (SERMs) like tamoxifen bind ER and antagonize the effects of estrogen on specific target genes $(8,36)$. Tamoxifen also has some estrogen agonist properties on certain genes and tissues, and augmentation of this property may play a role in resistance $(8,37)$. Estrogen deprivation is another mechanism utilized to antagonize ER (38). In premenopausal women, tamoxifen or pharmacological or surgical ovarian ablation are standard, while in postmenopausal women, 
aromatase inhibitors are prescribed to block the conversion of weak androgens of adrenal origin to estrogen in peripheral tissues as well as breast cancer tissue itself. Fulvestrant is an ER downregulator and a more potent antiestrogen that reduces ER levels in cells (39). Older endocrine therapies such as high-dose or physiological-dose estrogens and androgens work by lesser known mechanisms, although it has been proposed that high-dose estrogen can induce apoptosis by activation of the Fas ligand (40). Tamoxifen, but not estrogen deprivation or fulvestrant, activates the non-genomic ER, another property that could contribute to endocrine resistance in some cases.

\section{CLINICAL CLUES TO RESISTANCE TO ENDOCRINE THERAPY}

Several clinical observations provide clues to potential mechanisms for resistance to endocrine therapy (Table 1). ER loss over time in the tumor occurs in about $20 \%$ of patients treated with endocrine therapy (41-43). Such tumors would no longer be driven by estrogen, but the escape pathways which take over with loss of estrogen dependence have not been well defined. Upregulation of HER2 by either acquisition of gene amplification or overexpression has been shown to occur in some tumors (42, 44, 45). HER2 may subsequently assume the driving role in tumor progression by serving as an alternative survival pathway or by reducing the level of ER, thus rendering the tumor less responsive to estrogen $(25,46)$. Preclinical and clinical data suggest the possibility that tumors can alternate between ER and HER2 as the dominant pathway, with targeted therapy against one pathway causing reactivation of the other $(23,25,42,44,45,47-51)$. PR, on the other hand, is lost more frequently than ER with intervening endocrine therapy, and with this loss the tumor becomes more aggressive and patients have a worse survival outcome than patients who maintain PR expression after resistance to one endocrine therapy $(52,53)$. In this case, PR loss might be associated with increased growth factor signaling and upregulation of the PI3K pathway, which downregulates PR and ER expression $(27,54)$.

Response to one form of endocrine therapy after progression on another is a historically recognized observation that is the key to management of patients with metastatic disease. Tumors in such patients are still estrogen dependent but have become resistant to the ER targeted therapy being given. Responses to an aromatase inhibitor or fulvestrant after progression on tamoxifen are good examples of this phenomenon $(55,56)$. Subsequent responses to serial endocrine therapy tend to be shorter, coincident with a decline in ER level, suggesting a gradual shift from dependence on ER to an alternative escape pathway. High dose estrogen therapy was the first additive endocrine therapy for breast cancer that was replaced by tamoxifen and aromatase inhibitors due to their more favorable toxicity profile. It is important to note that patients responding and then progressing on high dose estrogen therapy frequently respond simply to estrogen withdrawal. Occasionally the disease can be controlled for many years by initiating and then sequencing high dose estrogen with estrogen withdrawal over time. One such patient of the author's with metastatic bone disease was controlled for over 8 years by alternating high-dose estrogen with estrogen withdrawal 3 separate times. Eventually all tumors completely lose estrogen dependence, even when ER is still expressed, by mechanisms which are poorly understood and are likely to be multiple as the tumor progresses to a more aggressive phenotype.

These observations suggest several types of endocrine therapy resistance (Table 2).

Resistance can take the form of de novo (existing before any treatment is given) or acquired (resistance that develops during a given therapy after an initial period of response). Some tumors lose estrogen dependence with loss of ER expression, although preclinical data suggest that ER can sometimes be reexpressed during subsequent treatment $(25,49,51)$. Other tumors lose estrogen dependence while still expressing ER, indicating that an escape pathway has developed to replace ER. Still other tumors continue to express ER but have 
not lost estrogen dependence and will respond to an alternative form of endocrine therapy. These tumors have developed resistance to the specific ER targeted therapy. The fact that subsequent remissions tend to be shorter and that ER levels decline over time suggests that other survival pathways are beginning to exert their effects or that an endocrine resistant clone is slowly emerging over time. The role that tumor cells with stem cell-like qualities play in the development of endocrine resistance, if any, remains to be clarified.

\section{SIGNALING MOLECULES AND PATHWAYS IMPLICATED IN RESISTANCE TO ENDOCRINE THERAPY}

Multiple pathways and molecules have been implicated in the diverse mechanisms responsible for endocrine resistance. These pathways and their gene networks, recently reviewed in (57), have mostly been investigated in the preclinical setting with a focus on tamoxifen. However, several alternative pathways have been shown or suggested to play a more general role in resistance to various other forms of endocrine therapy. Deregulation of these pathways most often arises from genetic or epigenetic changes in the tumor cells themselves. These changes influence uptake and metabolism of the endocrine agents and cellular responses to their inhibitory effects.

\section{Tumor Microenvironment and Host Associated Mechanisms of Resistance}

An emerging role for the tumor microenvironment as an important modulator of these processes and contributor to endocrine sensitivity has also been recognized in recent years. Evidence to support this notion has been substantiated from studies involving gene expression profiling and biomarkers associated with endocrine therapy responses $(58,59)$, and from more sophisticated in vitro and in vivo experimental model systems (35). Components of the microenvironment implicated in endocrine resistance include stromal (i) cells (e.g., fibroblasts, endothelial, and immune system cells), (ii) structural elements of the extracellular matrix (ECM), and (iii) soluble factors (e.g., growth factors and cytokines), as well as additional microenvironmental conditions, such as hypoxia and acidity (60). The role of tumor cell pathways engaged in mediating these microenvironmental and extracellular matrix stimuli, especially the integrin family and other adhesion molecules (e.g., CAS ${ }^{\mathrm{p} 130}$ ), has also recently been documented $(61,62)$, suggesting novel signaling axes (e.g., integrin/ FAK/SRC) that may be targeted to circumvent endocrine resistance. In addition, as a result of recent pharmacogenomic and high throughput studies, the list of additional host-genome associated factors governing endocrine sensitivity is also growing.

\section{Tumor Associated Mechanisms of Resistance}

As suggested above, however, most pathways potentially involved in endocrine resistance stem from the tumor cells themselves. These pathways fall broadly into three conceptual categories with shared and overlapping components and mechanisms.

ER and ER Coregulators-The first category consists of the ER itself, its coregulators, and additional factors that deregulate ER activity and modulate the receptor functions in response to endocrine therapy. As mentioned, loss of ER expression (i.e., the ERa isoform) in refractory endocrine tumors, though uncommon, results in an endocrine insensitive phenotype $(41,42)$. Importantly, however, therapies inhibiting growth factor receptor pathways known to downregulate ER can lead to restoration of ER expression and endocrine sensitivity in both preclinical and clinical settings $(25,49,51)$. The expression of ER splicing variants, specifically the newly identified short variant ERa36 (63) and estrogenrelated receptors (ERR), have also been implicated in reducing endocrine response. In addition, evidence indicates that negative (corepressors) and positive (coactivators) ER coregulators, which directly influence the balance of agonistic versus antagonistic activities 
of SERMs like tamoxifen and the ligand-independent activity of the ER, are critical in determining endocrine sensitivity and resistance (16 and references therein). Overexpression of the ER coactivator AIB1 (also known as SRC3 or NCOA3) is associated with clinical and experimental tamoxifen resistance $(12,14)$, while downregulation of the corepressor NCoR was documented in tamoxifen refractory experimental tumors (64). ER as well as its coregulators are also intimately regulated by posttranslational modifications (PTMs). Growth factor receptors (e.g., EGFR/HER2, IGF1-R, and FGFR) and additional cellular and stress-related kinases (e.g., AKT, p42/44, JNK, and p38 MAPKs, PKA, PAK-1, IKK, SRC, and CDK7) regulate multiple PTMs (16, 57, 65 and references therein). Phosphorylation, methylation, ubiquitination, and additional PTMs of ER and its coregulators have all been shown to influence ER activity and sensitivity to various endocrine therapies (57). ER can also reside outside the nucleus, engaging with cytoplasmic and membrane signaling complexes, and can activate and regulate various growth factor receptors and other cellular signaling pathways as a result $(13,14,31,32)$. Intriguingly, hyperactivation of these signaling pathways increases non-nuclear ER localization and its nongenomic activity, thus creating a positive feedback loop of crossactivation between the ER and growth factor receptor pathways. Importantly, this non-nuclear ER activity can be activated by both estrogen and tamoxifen, thus contributing to resistance $(13,14,32)$. Other endocrine therapies, however, such as the more potent ER degrader fulvestrant or strategies of estrogen deprivation, fail to trigger this nongenomic ER activity. Lastly, increased levels of transcription factors, such as NFkB and AP-1, that tether ER to specific gene promoters, have also been associated with endocrine resistance (66-68).

Cell Cycle Signaling Molecules-The second category of endocrine resistance-related pathways includes molecules involved in the cellular and biological responses to endocrine therapy such as inhibition of cell proliferation and induction of apoptosis. Most of the evidence for the role of these pathways stems from preclinical studies. As such, upregulation of positive regulators of the cell cycle, especially those controlling G1 phase progression, as well as downregulation of negative regulators of the cell cycle have been documented to interrupt and block the antiproliferative effects of endocrine therapy, leading to resistance $(57,69)$. For example, overexpression of the positive regulators MYC and cyclins E1 and D1 results in endocrine resistance either by activating cyclin-dependent kinases critical for G1 phase or by relieving the inhibitory effects of the negative cell cycle regulators p21 and p27 (69, 70). Likewise, reduced expression, stability, or activity of p21 and p27 (71, 72), as well as inactivation of the RB tumor suppressor, are also associated with poor response to endocrine therapy, especially tamoxifen. Of note, multiple growth factor receptors and their downstream signaling pathways, by modulating specific transcription factors, microRNAs, or by protein phosphorylation, downregulate expression or activity of these negative cell cycle regulators. Overexpression of HER2 and hyperactivation of AKT and SRC are prominent examples of such pathways. Consistent with the cytostatic effect of endocrine therapy, upregulation of cell survival signaling and anti-apoptotic molecules, such as BCL$\mathrm{X}_{\mathrm{L}}$, and decreases in expression of pro-apoptotic molecules, such as BIK and caspase 9, can also lead to endocrine resistance (73). As before, activation of growth factor receptor signaling via the PI3K/AKT pathway is important modulators of these apoptotic/survival molecules, but additional molecules such as NFkB have also been implicated (66). Finally, whether autophagy, recently shown to mediate cell survival, plays a more general role in endocrine resistance is yet to be determined (74).

Growth Factor Receptor Pathways-The third category of pathways involved in endocrine resistance are those that can provide alternative proliferation and survival stimuli to the tumors in the presence of effective inhibition of the ER pathway. Importantly, these pathways--such as growth factor and other cellular kinase pathways--can also circumvent 
the inhibitory effects of endocrine therapy via bidirectional crosstalk and modulation of the ER. However, many of these pathways can, either initially or eventually during the course of treatment, emerge to act as ER-independent drivers of tumor growth and survival, thus conferring a more global resistance phenotype to all types of endocrine therapy. Pathways such as the HER tyrosine kinase receptor family and receptors for insulin/IGF1, FGF, and VEGF, as well as cellular Src, AKT, and stress-related kinases have been implicated (7578). These pathways can be activated either by amplification and/or overexpression of the receptors or their cognate ligands. Pathway activation can also be achieved by deregulation of downstream signaling moieties, such as activating mutations in the PI3K catalytic subunit or loss of expression of the PTEN tumor suppressor of this pathway (79). The androgen receptor and potentially additional nuclear receptors have also been implicated as alternative growth-stimulators that can bypass ER inhibition and lead to resistance (80). Importantly, while these pathways and mechanisms are varied and span a wide range of signaling cascades and gene networks, EGFR and HER2 have been recognized as one of the most prominent factors contributing to endocrine resistance. As a result, many clinical strategies have focused on co-targeting this pathway together with ER to circumvent endocrine resistance and improve patient outcome.

\section{CLINICAL TRIALS DESIGNED TO OVERCOME ENDOCRINE RESISTANCE}

Many clinical trials have begun to test the idea that growth factor receptor signaling contributes to de novo or acquired endocrine resistance $(75,81,82)$. Some of these trials were short term neoadjuvant trials, some phase II, some randomized phase II and some phase III in patients with metastatic disease. Some focused on HER2 positive patients while others included all ER positive patients, regardless of HER2 status.

The Tandem trial randomized patients with HER2 positive tumors to arimidex alone or arimdex plus trastuzumab (83). The results clearly showed an advantage for the combination, although both arms did poorly, thereby exemplifying the difficulty in controlling ER positive/HER2+ disease. Despite targeting both major pathways, remissions are few and brief due to the rapid development of more dominant survival pathways in metastatic disease.

Because preclinical studies suggest a major role for the EGFR (HER1) in acquired endocrine resistance, many trials incorporated gefitinib into the endocrine therapy regimen with tamoxifen or an aromatase inhibitor (23). Some of these studies have not yet been published but have been presented at meetings and published as abstracts. Two randomized Phase II trials of somewhat similar design randomized patients with ER+ metastatic breast cancer to either tamoxifen $+/$ - gefitinib or to anastrozole $+/$ - gefitinib $(84,85)$. One of these trials was terminated early because of slow patient accrual, but it showed a numerical advantage in clinical benefit rate and progression free survival (PFS) with the addition of gefitinib to anastrozole (85). The other larger trial with 290 patients also showed a numerical advantage in favor of gefitinib added to tamoxifin (84). Both trials reported that the advantage was confined to previously untreated patients and suggested that an advantage was seen even in patients whose tumors were initially negative for HER2 overexpression $(84,85)$. These studies concluded that the strategy of combining an EGFR inhibitor with an endocrine agent was of sufficient interest to warrant further study and that studies designed to select the appropriate patients for combined treatment were paramount. A much smaller trial in more heavily treated patients failed to confirm these data and showed a high rate of patient withdrawal from the study (30\%) due to side effects of gefitinib (86). Finally, another trial comparing anastrozole plus gefitinib with fulvestrant plus gefitinib showed that the regimens were tolerable and suggested a slight advantage for the anastrozole combination (87). 
The largest trial by far (1286 patients) compared letrozole with or without lapatinib in patients with ER-positive metastatic breast cancer (88). This trial showed a significant advantage in PFS and response rate in the HER2 positive subset for the addition of lapatinib. In the HER2 negative subset, there was no overall significant benefit from lapatinib, but a pre-planned Cox regression analysis showed a $23 \%$ reduction in the risk of progression with the addition of lapatinib. The benefit was seen in those who had discontinued tamoxifen therapy within 6 months of entering the study. A more recent analysis showed that patients with tumors exhibiting lower ER levels received the most benefit (89). This result, which will require confirmation from other trials, is consistent with data suggesting that growth factor signaling downregulates ER expression $(3,25)$. Perhaps those tumors with lower ER expression are also those which rely on the HER pathway when ER is blocked by endocrine therapy. These tumors would be expected to respond well to a HER inhibitor.

Two randomized neoadjuvant studies have been reported comparing anastrozole plus gefitinib $(90,91)$. One small study in patients selected for higher expression of EGFR evaluated gefitinib alone with gefitinib plus anastrozole (91). Both treatment regimens reduced phosphorylation of EGFR, Ki67 index, and tumor size, suggesting that gefitinib is effective in tumors selected by EGFR expression. The other study compared anastrozole alone with anastrozole plus gefitinib in patients selected only by ER status (90). If EGFR expression is important for the response to gefitinib, and if expression rises over time in patients treated with endocrine therapy, then the neoadjuvant setting, where EGFR levels would be low, may not be optimal to investigate this new strategy. Like the preclinical studies that led to testing this strategy in patients (23), gefitinib would be expected to delay the onset of acquired resistance but exert a minimal impact on initial response. In fact, this neoadjuvant trial showed no benefit from gefitinib, and, in fact, showed a trend for a reduced antitumor effect on Ki67 and tumor response with the combination (90).

Other trials in metastatic disease evaluated inhibitors of signaling molecules downstream from HER receptors. Two trials of mTOR inhibitors have been reported in combination with endocrine therapy $(92,93)$. A randomized Phase II study compared letrozole alone with letrozole plus temsirolimus in hormone receptor-positive metastatic breast cancer and suggested a benefit. The trial was expanded to a Phase III trial that was terminated early due to lack of efficacy of the combination (92). The Phase III portion of the study used a lower dose than the Phase II portion, due to toxicity with the higher dose. Another randomized Phase II trial of letrozole with or without everolimus using an optimal dose of the inhibitor in the neoadjuvant setting showed a statistically significant increase in response with the combination (93).

\section{CONCLUSIONS}

ER targeted therapy has improved the quality of life and survival of millions of women around the world in the past 3 decades, but resistance to therapy continues to be a major problem. The ER signaling pathway is a complex network with many levels of control including extensive crosstalk with growth factor signaling pathways, thus offering several possible mechanisms of resistance. Clinical trials in patients suggest that HER2 overexpressing, ER positive breast cancers should be treated with a combination of ERtargeted and HER-targeted therapies. Early results from clinical trials also suggest that subsets of patients with ER+, HER2 negative breast cancers may benefit from a combination of a growth factor pathway inhibitor with ER-targeted therapy such as tamoxifen or an aromatase inhibitor. Further studies are needed to confirm and expand these observations and to identify a priori those patients most likely to benefit from this approach. Finally, ongoing and planned additional studies combining ER-targeted and growth factor pathwaytargeted therapy will determine whether this strategy is of value. 
It is likely that there are many causes of resistance to endocrine therapy once a tumor becomes independent of estrogen. One factor limiting our understanding of these varied mechanisms is the lack of tumor tissue for detailed studies before treatment and after resistance has developed. This type of study will be crucial if we are to learn which escape pathways become activated in endocrine resistance and which targeted therapies can prevent or overcome this type of tumor progression.

\section{Acknowledgments}

Grant Support: This work was supported in part by National Cancer Institute (NCI) grants P30CA125123 and P50CA058183, and funding from the Stand Up to Cancer-Breast Cancer Dream Team

\section{Acronyms}

$\begin{array}{ll}\text { ER } & \text { estrogen receptor (alpha) } \\ \text { PR } & \text { progesterone receptor } \\ \text { EGFR } & \text { epidermal growth factor receptor } \\ \text { HER2 } & \text { human epidermal growth factor receptor 2 } \\ \text { IGF1-R } & \text { insulin-like growth factor receptor } \\ \text { PI3K } & \text { Phosphoinositide 3-kinase } \\ \text { MAPKs } & \text { mitogen-activated protein kinases } \\ \text { AIB1/SRC-3 } & \text { amplified in breast cancer-1/steroid receptor coactivator } \\ \text { PTM } & \text { posttranslational modifications } \\ \text { PFS } & \text { progression free survival }\end{array}$

\section{References}

1. Clark GM, Osborne CK, McGuire WL. Correlations between estrogen receptor, progesterone receptor, and patient characteristics in human breast cancer. J Clin Oncol. 1984; 2:1102-9. [PubMed: 6491696]

2. Wenger CR, Beardslee S, Owens MA, et al. DNA ploidy, S-phase, and steroid receptors in more than 127,000 breast cancer patients. Breast Cancer Res Treat. 1993; 28:9-20. [PubMed: 8123871]

3. Schiff, R.; Osborne, CK.; Fuqua, SA. Clinical Aspects of Estrogen and Progesterone Receptors. In: Harris, JR.; Lippman, ME.; Morrow, M.; Osborne, CK., editors. Diseases of the Breast 4th Edition. Philadelphia, PA: Wolters Kluwer/Lippincott Williams \& Wilkins; 2009. p. 408-30.

4. Paik S, Shak S, Tang G, et al. A multigene assay to predict recurrence of tamoxifen-treated, nodenegative breast cancer. N Engl J Med. 2004; 351:2817-26. [PubMed: 15591335]

5. van de Vijver MJ, He YD, van't Veer LJ, et al. A gene-expression signature as a predictor of survival in breast cancer. N Engl J Med. 2002; 347:1999-2009. [PubMed: 12490681]

6. Baum M, Budzar AU, Cuzick J, et al. Anastrozole alone or in combination with tamoxifen versus tamoxifen alone for adjuvant treatment of postmenopausal women with early breast cancer: first results of the ATAC randomised trial. Lancet. 2002; 359:2131-9. [PubMed: 12090977]

7. Coates AS, Keshaviah A, Thurlimann B, et al. Five years of letrozole compared with tamoxifen as initial adjuvant therapy for postmenopausal women with endocrine-responsive early breast cancer: update of study BIG 1-98. J Clin Oncol. 2007; 25:486-92. [PubMed: 17200148]

8. Frasor J, Stossi F, Danes JM, et al. Selective estrogen receptor modulators: discrimination of agonistic versus antagonistic activities by gene expression profiling in breast cancer cells. Cancer Res. 2004; 64:1522-33. [PubMed: 14973112]

9. Klinge CM. Estrogen receptor interaction with estrogen response elements. Nucleic Acids Res. 2001; 29:2905-19. [PubMed: 11452016] 
10. Kushner PJ, Agard DA, Greene GL, et al. Estrogen receptor pathways to AP-1. J Steroid Biochem Mol Biol. 2000; 74:311-7. [PubMed: 11162939]

11. Smith CL, O'Malley BW. Coregulator function: a key to understanding tissue specificity of selective receptor modulators. Endocr Rev. 2004; 25:45-71. [PubMed: 14769827]

12. Osborne CK, Bardou V, Hopp TA, et al. Role of the estrogen receptor coactivator AIB1 (SRC-3) and HER-2/neu in tamoxifen resistance in breast cancer. J Natl Cancer Inst. 2003; 95:353-61. [PubMed: 12618500]

13. Schiff R, Massarweh SA, Shou J, et al. Cross-talk between estrogen receptor and growth factor pathways as a molecular target for overcoming endocrine resistance. Clin Cancer Res. 2004; 10:331S-6S. [PubMed: 14734488]

14. Shou J, Massarweh S, Osborne CK, et al. Mechanisms of tamoxifen resistance: increased estrogen receptor-HER2/neu cross-talk in ER/HER2-positive breast cancer. J Natl Cancer Inst. 2004; 96:926-35. [PubMed: 15199112]

15. Wu RC, Smith CL, O’Malley BW. Transcriptional regulation by steroid receptor coactivator phosphorylation. Endocr Rev. 2005; 26:393-9. [PubMed: 15814849]

16. Schiff R, Massarweh S, Shou J, Osborne CK. Breast cancer endocrine resistance: how growth factor signaling and estrogen receptor coregulators modulate response. Clin Cancer Res. 2003; 9:447S-54S. [PubMed: 12538499]

17. Vyhlidal C, Samudio I, Kladde MP, Safe S. Transcriptional activation of transforming growth factor alpha by estradiol: requirement for both a GC-rich site and an estrogen response element half-site. J Mol Endocrinol. 2000; 24:329-38. [PubMed: 10828826]

18. Lee AV, Cui X, Oesterreich S. Cross-talk among estrogen receptor, epidermal growth factor, and insulin-like growth factor signaling in breast cancer. Clin Cancer Res. 2001; 7:4429s-35s. (Abstr.) discussion 11s-12s. [PubMed: 11916236]

19. Umayahara Y, Kawamori R, Watada H, et al. Estrogen regulation of the insulin-like growth factor I gene transcription involves an AP-1 enhancer. J Biol Chem. 1994; 269:16433-42. [PubMed: 8206951]

20. Nicholson RI, Hutcheson IR, Hiscox SE, et al. Growth factor signalling and resistance to selective oestrogen receptor modulators and pure anti-oestrogens: the use of anti-growth factor therapies to treat or delay endocrine resistance in breast cancer. Endocr Relat Cancer. 2005; 12(Suppl 1):S2936. [PubMed: 16113097]

21. Yarden RI, Wilson MA, Chrysogelos SA. Estrogen suppression of EGFR expression in breast cancer cells: a possible mechanism to modulate growth. J Cell Biochem Suppl. 2001; 36:232-46. [PubMed: 11455588]

22. Newman SP, Bates NP, Vernimmen D, et al. Cofactor competition between the ligand-bound oestrogen receptor and an intron 1 enhancer leads to oestrogen repression of ERBB2 expression in breast cancer. Oncogene. 2000; 19:490-7. [PubMed: 10698518]

23. Massarweh S, Osborne CK, Creighton CJ, et al. Tamoxifen resistance in breast tumors is driven by growth factor receptor signaling with repression of classic estrogen receptor genomic function. Cancer Res. 2008; 68:826-33. [PubMed: 18245484]

24. Bayliss J, Hilger A, Vishnu P, et al. Reversal of the estrogen receptor negative phenotype in breast cancer and restoration of antiestrogen response. Clin Cancer Res. 2007; 13:7029-36. [PubMed: 18056179]

25. Lopez-Tarruella S, Schiff R. The dynamics of estrogen receptor status in breast cancer: re-shaping the paradigm. Clin Cancer Res. 2007; 13:6921-5. [PubMed: 18056165]

26. Cui X, Zhang P, Deng W, et al. Insulin-like growth factor-I inhibits progesterone receptor expression in breast cancer cells via the phosphatidylinositol 3-kinase/Akt/mammalian target of rapamycin pathway: progesterone receptor as a potential indicator of growth factor activity in breast cancer. Mol Endocrinol. 2003; 17:575-88. [PubMed: 12554765]

27. Cui X, Schiff R, Arpino G, et al. Biology of progesterone receptor loss in breast cancer and its implications for endocrine therapy. J Clin Oncol. 2005; 23:7721-35. [PubMed: 16234531]

28. Guo S, Sonenshein GE. Forkhead box transcription factor FOXO3a regulates estrogen receptor alpha expression and is repressed by the Her-2/neu/phosphatidylinositol 3-kinase/Akt signaling pathway. Mol Cell Biol. 2004; 24:8681-90. [PubMed: 15367686] 
29. Creighton CJ, Fu X, Hennessy BT, et al. Proteomic and transcriptomic profiling reveals a link between the PI3K pathway and lower estrogen receptor (ER) levels and activity in ER+ breast cancer. Breast Cancer Res. 2010 Accepted.

30. Brinkman JA, El-Ashry D. ER re-expression and re-sensitization to endocrine therapies in ERnegative breast cancers. J Mammary Gland Biol Neoplasia. 2009; 14:67-78. [PubMed: 19263197]

31. Levin ER, Pietras RJ. Estrogen receptors outside the nucleus in breast cancer. Breast Cancer Res Treat. 2008; 108:351-61. [PubMed: 17592774]

32. Santen RJ, Fan P, Zhang Z, et al. Estrogen signals via an extra-nuclear pathway involving IGF-1R and EGFR in tamoxifen-sensitive and -resistant breast cancer cells. Steroids. 2009; 74:586-94. [PubMed: 19138696]

33. Wu RC, Qin J, Yi P, et al. Selective phosphorylations of the SRC-3/AIB1 coactivator integrate genomic reponses to multiple cellular signaling pathways. Mol Cell. 2004; 15:937-49. [PubMed: 15383283]

34. Lee H, Bai W. Regulation of estrogen receptor nuclear export by ligand-induced and p38-mediated receptor phosphorylation. Mol Cell Biol. 2002; 22:5835-45. [PubMed: 12138194]

35. Pontiggia O, Rodriguez V, Fabris V, et al. Establishment of an in vitro estrogen-dependent mouse mammary tumor model: a new tool to understand estrogen responsiveness and development of tamoxifen resistance in the context of stromal-epithelial interactions. Breast Cancer Res Treat. 2009; 116:247-55. [PubMed: 18622696]

36. Jordan VC. The science of selective estrogen receptor modulators: concept to clinical practice. Clin Cancer Res. 2006; 12:5010-3. [PubMed: 16951214]

37. Jordan VC, O'Malley BW. Selective estrogen-receptor modulators and antihormonal resistance in breast cancer. J Clin Oncol. 2007; 25:5815-24. [PubMed: 17893378]

38. Jordan VC, Brodie AM. Development and evolution of therapies targeted to the estrogen receptor for the treatment and prevention of breast cancer. Steroids. 2007; 72:7-25. [PubMed: 17169390]

39. Dowsett M, Nicholson RI, Pietras RJ. Biological characteristics of the pure antiestrogen fulvestrant: overcoming endocrine resistance. Breast Cancer Res Treat. 2005; 93(Suppl 1):S11-8. [PubMed: 16247595]

40. Lewis-Wambi JS, Jordan VC. Estrogen regulation of apoptosis: how can one hormone stimulate and inhibit? Breast Cancer Res. 2009; 11:206. [PubMed: 19519952]

41. Encarnacion CA, Ciocca DR, McGuire WL, et al. Measurement of steroid hormone receptors in breast cancer patients on tamoxifen. Breast Cancer Res Treat. 1993; 26:237-46. [PubMed: 8251648]

42. Gutierrez MC, Detre S, Johnston S, et al. Molecular changes in tamoxifen-resistant breast cancer: relationship between estrogen receptor, HER-2, and p38 mitogen-activated protein kinase. J Clin Oncol. 2005; 23:2469-76. [PubMed: 15753463]

43. Hull DF 3rd, Clark GM, Osborne CK, et al. Multiple estrogen receptor assays in human breast cancer. Cancer Res. 1983; 43:413-6. [PubMed: 6847780]

44. Lipton A, Leitzel K, Ali SM, et al. Serum HER-2/neu conversion to positive at the time of disease progression in patients with breast carcinoma on hormone therapy. Cancer. 2005; 104:257-63. [PubMed: 15952182]

45. Meng S, Tripathy D, Shete S, et al. HER-2 gene amplification can be acquired as breast cancer progresses. Proc Natl Acad Sci U S A. 2004; 101:9393-8. [PubMed: 15194824]

46. Arpino G, Green SJ, Allred DC, et al. HER-2 amplification, HER-1 expression, and tamoxifen response in estrogen receptor-positive metastatic breast cancer: a southwest oncology group study. Clin Cancer Res. 2004; 10:5670-6. [PubMed: 15355892]

47. Massarweh S, Osborne CK, Jiang S, et al. Mechanisms of tumor regression and resistance to estrogen deprivation and fulvestrant in a model of estrogen receptor-positive, HER-2/neu-positive breast cancer. Cancer Res. 2006; 66:8266-73. [PubMed: 16912207]

48. Creighton CJ, Massarweh S, Huang S, et al. Development of resistance to targeted therapies transforms the clinically associated molecular profile subtype of breast tumor xenografts. Cancer Res. 2008; 68:7493-501. [PubMed: 18794137] 
49. Munzone E, Curigliano G, Rocca A, et al. Reverting estrogen-receptor-negative phenotype in HER-2-overexpressing advanced breast cancer patients exposed to trastuzumab plus chemotherapy. Breast Cancer Res. 2006; 8:R4. [PubMed: 16417653]

50. Reid G, Denger S, Kos M, Gannon F. Human estrogen receptor-alpha: regulation by synthesis, modification and degradation. Cell Mol Life Sci. 2002; 59:821-31. [PubMed: 12088282]

51. Xia W, Bacus S, Hegde P, et al. A model of acquired autoresistance to a potent ErbB2 tyrosine kinase inhibitor and a therapeutic strategy to prevent its onset in breast cancer. Proc Natl Acad Sci U S A. 2006; 103:7795-800. [PubMed: 16682622]

52. Brankovic-Magic M, Jankovic R, Neskovic-Konstantinovic Z, Nikolic-Vukosavljevic D. Progesterone receptor status of breast cancer metastases. J Cancer Res Clin Oncol. 2002; 128:5560. [PubMed: 11862473]

53. Gross GE, Clark GM, Chamness GC, McGuire WL. Multiple progesterone receptor assays in human breast cancer. Cancer Res. 1984; 44:836-40. [PubMed: 6692382]

54. Arpino G, Weiss H, Lee AV, et al. Estrogen receptor-positive, progesterone receptor-negative breast cancer: association with growth factor receptor expression and tamoxifen resistance. J Natl Cancer Inst. 2005; 97:1254-61. [PubMed: 16145046]

55. Dodwell D, Wardley A, Johnston S. Postmenopausal advanced breast cancer: options for therapy after tamoxifen and aromatase inhibitors. Breast. 2006; 15:584-94. [PubMed: 16504510]

56. Robertson JF, Osborne CK, Howell A, et al. Fulvestrant versus anastrozole for the treatment of advanced breast carcinoma in postmenopausal women: a prospective combined analysis of two multicenter trials. Cancer. 2003; 98:229-38. [PubMed: 12872340]

57. Musgrove EA, Sutherland RL. Biological determinants of endocrine resistance in breast cancer. Nat Rev Cancer. 2009; 9:631-43. [PubMed: 19701242]

58. Helleman J, Jansen MP, Ruigrok-Ritstier K, et al. Association of an extracellular matrix gene cluster with breast cancer prognosis and endocrine therapy response. Clin Cancer Res. 2008; 14:5555-64. [PubMed: 18765548]

59. Jansen MP, Foekens JA, van Staveren IL, et al. Molecular classification of tamoxifen-resistant breast carcinomas by gene expression profiling. J Clin Oncol. 2005; 23:732-40. [PubMed: 15681518]

60. Generali D, Berruti A, Brizzi MP, et al. Hypoxia-inducible factor-1alpha expression predicts a poor response to primary chemoendocrine therapy and disease-free survival in primary human breast cancer. Clin Cancer Res. 2006; 12:4562-8. [PubMed: 16899602]

61. Folgiero V, Avetrani P, Bon G, et al. Induction of ErbB-3 expression by alpha6beta4 integrin contributes to tamoxifen resistance in ERbeta1-negative breast carcinomas. PLoS One. 2008; 3:e1592. [PubMed: 18270579]

62. Brinkman A, de Jong D, Tuinman S, et al. The substrate domain of BCAR1 is essential for antiestrogen-resistant proliferation of human breast cancer cells. Breast Cancer Res Treat. 2010; 120:401-8. [PubMed: 19412734]

63. Shi L, Dong B, Li Z, et al. Expression of ER-a36, a novel variant of estrogen receptor a, and resistance to tamoxifen treatment in breast cancer. J Clin Oncol. 2009; 27:3423-9. [PubMed: 19487384]

64. Lavinsky RM, Jepsen K, Heinzel T, et al. Diverse signaling pathways modulate nuclear receptor recruitment of N-CoR and SMRT complexes. Proc Natl Acad Sci U S A. 1998; 95:2920-5. [PubMed: 9501191]

65. Ali S, Coombes RC. Endocrine-responsive breast cancer and strategies for combating resistance. Nat Rev Cancer. 2002; 2:101-12. [PubMed: 12635173]

66. Zhou Y, Yau C, Gray JW, et al. Enhanced NF kappa B and AP-1 transcriptional activity associated with antiestrogen resistant breast cancer. BMC Cancer. 2007; 7:59. [PubMed: 17407600]

67. Johnston SR, Lu B, Scott GK, et al. Increased activator protein-1 DNA binding and c-Jun NH2terminal kinase activity in human breast tumors with acquired tamoxifen resistance. Clin Cancer Res. 1999; 5:251-6. [PubMed: 10037172]

68. Schiff R, Reddy P, Ahotupa M, et al. Oxidative stress and AP-1 activity in tamoxifen-resistant breast tumors in vivo. J Natl Cancer Inst. 2000; 92:1926-34. [PubMed: 11106684] 
69. Span PN, Tjan-Heijnen VC, Manders P, et al. Cyclin-E is a strong predictor of endocrine therapy failure in human breast cancer. Oncogene. 2003; 22:4898-904. [PubMed: 12894232]

70. Butt AJ, McNeil CM, Musgrove EA, Sutherland RL. Downstream targets of growth factor and oestrogen signalling and endocrine resistance: the potential roles of c-Myc, cyclin D1 and cyclin E. Endocr Relat Cancer. 2005; 12(Suppl 1):S47-59. [PubMed: 16113099]

71. Chu IM, Hengst L, Slingerland JM. The Cdk inhibitor p27 in human cancer: prognostic potential and relevance to anticancer therapy. Nat Rev Cancer. 2008; 8:253-67. [PubMed: 18354415]

72. Perez-Tenorio G, Berglund F, Esguerra Merca A, et al. Cytoplasmic p21WAF1/CIP1 correlates with Akt activation and poor response to tamoxifen in breast cancer. Int J Oncol. 2006; 28:103142. [PubMed: 16596219]

73. Riggins RB, Bouton AH, Liu MC, Clarke R. Antiestrogens, aromatase inhibitors, and apoptosis in breast cancer. Vitam Horm. 2005; 71:201-37. [PubMed: 16112269]

74. Schoenlein PV, Periyasamy-Thandavan S, Samaddar JS, et al. Autophagy facilitates the progression of ERalpha-positive breast cancer cells to antiestrogen resistance. Autophagy. 2009; 5:400-3. [PubMed: 19221464]

75. Arpino G, Wiechmann L, Osborne CK, Schiff R. Crosstalk between the estrogen receptor and the HER tyrosine kinase receptor family: molecular mechanism and clinical implications for endocrine therapy resistance. Endocr Rev. 2008; 29:217-33. [PubMed: 18216219]

76. Chakraborty AK, Welsh A, Digiovanna MP. Co-targeting the insulin-like growth factor I receptor enhances growth-inhibitory and pro-apoptotic effects of anti-estrogens in human breast cancer cell lines. Breast Cancer Res Treat. 2010; 120:327-35. [PubMed: 19337828]

77. Kern FG, McLeskey SW, Zhang L, et al. Transfected MCF-7 cells as a model for breast-cancer progression. Breast Cancer Res Treat. 1994; 31:153-65. [PubMed: 7881095]

78. Morgan L, Gee J, Pumford S, et al. Elevated Src kinase activity attenuates Tamoxifen response in vitro and is associated with poor prognosis clinically. Cancer Biol Ther. 2009; 8:1550-8. [PubMed: 19830888]

79. Shoman N, Klassen S, McFadden A, et al. Reduced PTEN expression predicts relapse in patients with breast carcinoma treated by tamoxifen. Mod Pathol. 2005; 18:250-9. [PubMed: 15475931]

80. De Amicis F, Thirugnansampanthan J, Cui Y, et al. Androgen receptor overexpression induces tamoxifen resistance in human breast cancer cells. Breast Cancer Res Treat. 2009; 121:1-11. [PubMed: 19533338]

81. Johnston SR. New strategies in estrogen receptor-positive breast cancer. Clin Cancer Res. 2010; 16:1979-87. [PubMed: 20332324]

82. Johnston SR. Clinical efforts to combine endocrine agents with targeted therapies against epidermal growth factor receptor/human epidermal growth factor receptor 2 and mammalian target of rapamycin in breast cancer. Clin Cancer Res. 2006; 12:1061s-68s. [PubMed: 16467125]

83. Kaufman B, Mackey JR, Clemens MR, et al. Trastuzumab plus anastrozole versus anastrozole alone for the treatment of postmenopausal women with human epidermal growth factor receptor 2positive, hormone receptor-positive metastatic breast cancer: results from the randomized phase III TAnDEM study. J Clin Oncol. 2009; 27:5529-37. [PubMed: 19786670]

84. Osborne K, Neven P, Dirix L, et al. Randomized Phase II study of gefitinib (IRESSA) or placebo in combination with tamoxifen in patients with hormone receptor positive metastatic breast cancer. Breast Cancer Res Treat. 2007; 106:S1-S107. (Abstr. 2067). [PubMed: 17957466]

85. Cristofanilli M, Valero V, Mangalik A, et al. Phase II, randomized trial to compare anastrozole combined with gefitinib or placebo in postmenopausal women with hormone receptor-positive metastatic breast cancer. Clin Cancer Res. 2010; 16:1904-14. [PubMed: 20215537]

86. Mauriac, L.; Cameron, D.; Dirix, L., et al. Results of randomized phase II trial combining Iressa ${ }^{\circledR}$ (gefitinib) and arimidex in women with advanced breast cancer (ABC). EORTC protocol 10021. Cancer Res; Presented at San Antonio Bareast Cancer Symposium, 31st; San Antonio, TX. 2009. (Abstr. 6133)

87. Carlson, RW.; O’Neill, A.; Vidaurre, T., et al. Randomized phase II trial of gefitinib plus anastrozole or fulvestrant in postmenopausal, metastatic breast cancer. J Clin Oncol; Presented at Annu. Meet. Am. Society of Clinical Oncology, 45th; Orlando. 2009. p. 15s(Abstr. 1013) 
88. Johnston S, Pippen J Jr, Pivot X, et al. Lapatinib combined with letrozole versus letrozole and placebo as first-line therapy for postmenopausal hormone receptor-positive metastatic breast cancer. J Clin Oncol. 2009; 27:5538-46. [PubMed: 19786658]

89. Finn, RS.; Press, M.; Dering, J., et al. Progression-free survival (PFS) of patients with HER2negative, estrogen-receptor (ER)-low metastatic breast cancer (MBC) with the addition of lapatinib to letrozole: Biomarker results of EGF30008. J Clin Oncol; Presented at Annu. Meet. Am. Society of Clinical Oncology, 45th; Orlando. 2009. p. 15s(Abstr. 1018)

90. Smith IE, Walsh G, Skene A, et al. A phase II placebo-controlled trial of neoadjuvant anastrozole alone or with gefitinib in early breast cancer. J Clin Oncol. 2007; 25:3816-22. [PubMed: 17679728]

91. Polychronis A, Sinnett HD, Hadjiminas D, et al. Preoperative gefitinib versus gefitinib and anastrozole in postmenopausal patients with oestrogen-receptor positive and epidermal-growthfactor-receptor-positive primary breast cancer: a double-blind placebo-controlled phase II randomised trial. Lancet Oncol. 2005; 6:383-91. [PubMed: 15925816]

92. Chow L, Sun Y, Jassem J, et al. Phase 3 study of temsirolimus with letrozole or letrozole alone in postmenopausal women with locally advanced or metastatic breast cancer. Breast Cancer Res Treat. 2006; 100(Suppl 1):S28. (Abstr. 6091).

93. Baselga J, Semiglazov V, van Dam P, et al. Phase II randomized study of neoadjuvant everolimus plus letrozole compared with placebo plus letrozole in patients with estrogen receptor-positive breast cancer. J Clin Oncol. 2009; 27:2630-7. [PubMed: 19380449] 


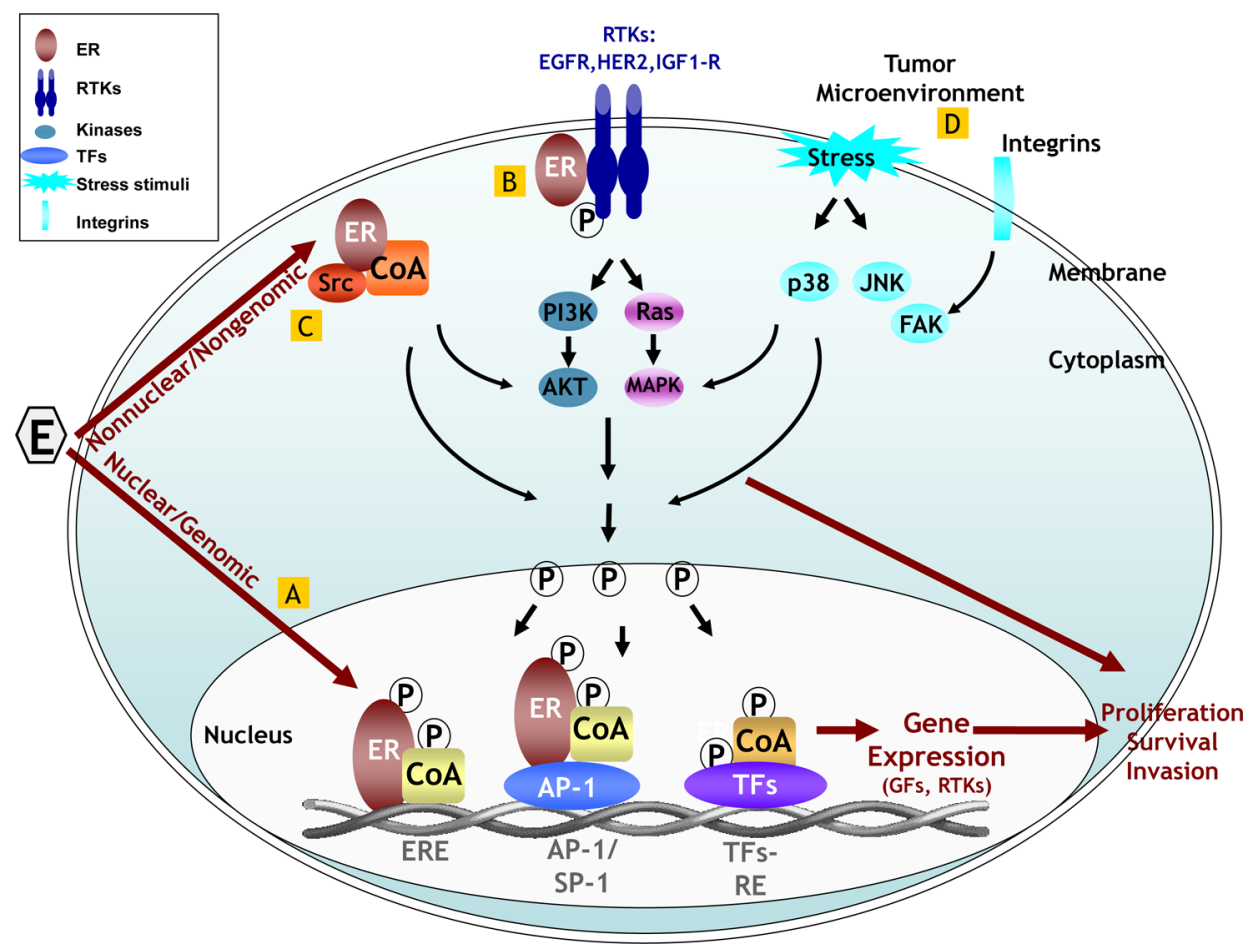

Figure 1. Mechanisms of Estrogen Receptor (ER) Action in Breast Cancer

Estrogen (E)-bound ER, acting as a transcription factor in the nucleus (nuclear/genomic activity), binds to DNA sequences in promoter regions of target genes either directly (at estrogen receptor elements; EREs) or indirectly via protein-protein interaction with other transcription factors at their cognate DNA responsive sites (e.g., members of the AP-1 or the SP-1 transcription complexes at AP-1 or SP-1 sites). Upon estrogen binding, ER generally recruits coactivator complexes $(\mathrm{CoA})$ to induce or modulate gene transcription including genes coding growth factors (GFs) and receptor tyrosine kinases (RTKs) (A). A small subset of the cellular pool of ER localized outside the nucleus and/or at the cell membrane associates in response to estrogen with growth factor RTKs (e.g., EGFR, HER2, and IGF1R) (B) and with additional signaling and coactivator molecules (e.g. the Src kinase) (C). This interaction, similar to GF activation of these pathways, activates multiple downstream kinase pathways (e.g., Src, PI3K/AKT, and Ras/p42/44 MAPK) which in turn phosphorylate various transcription factors (TFs) and coregulators, including components of the ER pathway that enhance gene expression on EREs and other response elements (RE). The nonnuclear/nongenomic activity, which can also be activated by tamoxifen, is enhanced in the presence of overexpression and hyperactivation of RTKs and can contribute to endocrine therapy resistance. Overall, the nuclear/genomic and nonnuclear/nogenomic ER activities work in concert to provide breast tumor cells with proliferation, survival and invasion stimuli. Signaling from the microenvironment activates stress related pathways and members of the integrin family. These pathways then trigger downstream kinase pathways (e.g., FAK, JNK and p38 MAPK) that can further modulate components of the transcriptional machinery, including ER (D). Alterations in each of these transcriptional and signaling elements can mediate resistance to endocrine therapy either by modulating ER activity or, by acting as escape pathways to provide alternative proliferation and survival stimuli. 
Table 1

Clinical Clues to Mechanisms of Resistance to Endocrine Therapy

\begin{tabular}{|l|}
\hline Decrease or loss of ER \\
\hline Upregulation of HER2 in some patients after endocrine therapy \\
\hline Loss of PR after progression on endocrine therapy \\
\hline Response to sequential endocrine therapies \\
\hline Shorter response duration and less frequent responses with sequential endocrine therapies \\
\hline Withdrawal response after high dose estrogen therapy \\
\hline Eventual loss of dependence on estrogen with resistance to all endocrine therapies \\
\hline Lower clinical benefit to endocrine therapy in high grade, highly proliferating, high 21-gene profile scores, ER positive tumors. \\
\hline
\end{tabular}


Table 2

Types of Endocrine Therapy Resistance

\begin{tabular}{|l|}
\hline De novo \\
\hline Acquired during treatment \\
\hline Loss of estrogen dependence due to loss of ER \\
\hline Loss of estrogen dependence despite presence of ER \\
\hline Resistance to a specific therapy; tumor still estrogen dependent \\
\hline
\end{tabular}

